\title{
A RAILROAD INTERMODAL CAPACITY MODEL
}

\author{
Michael L. Weigel \\ Union Pacific Railroad \\ 1416 Dodge Street \\ Omaha, NE 68179, U.S.A.
}

\begin{abstract}
This paper reports on the use of discrete event simulation to model the operations at Railroad Intermodal facilities. This paper will discuss the approach used at Union Pacific Railroad to develop a flexible capacity planning model. This paper will provide details on the processes modeled and the method used to reproduce the important characteristics of the actual system.
\end{abstract}

\section{INTRODUCTION}

With the rapid growth in Intermodal traffic many managers are seeking answers to questions concerning capacity. This paper presents a description of a general-purpose model of a Railroad Intermodal facility. The model is used to identify "weak links" in the system and provide a capacity estimate for planning capital improvements. Capacity estimates are determined by looking at the affects of train schedules, facility design and equipment availability on terminal performance.

\section{INTERMODAL SYSTEM OVERVIEW}

Railroad intermodal terminals are the interchange points between railroad and highway modes of transportation. The purpose of the intermodal terminal is to transfer freight loaded in containers or trailers (boxes) on and off railcars. It also provides interim storage for inbound loads waiting for customer pickup.

The equipment used at the terminal include: packers or cranes that lift arriving or departing loads to and from the railcars, and ramp tractors for moving loads between the loading/unloading pads and parking lots.

Trailer and containers arriving at the terminal go through a gate check-in process. The gate operators check for waybill information and inspect the box for damage. Once through the gate the boxes are moved to track side for loading or left in a outbound staging lot.
Track side placement is based on the destination of the trailer or container. The container will then be loaded onto the railcar by a side loading packer or top loading crane.

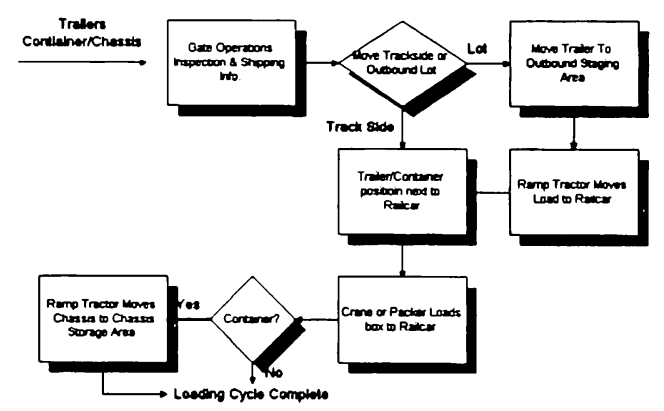

Figure 1: Loading Process

Intermodal trains arriving at the terminal will be unloaded and the trailers or containers moved by ramp tractors to a designated parking area. The load remains on the facility until picked up by the customer.

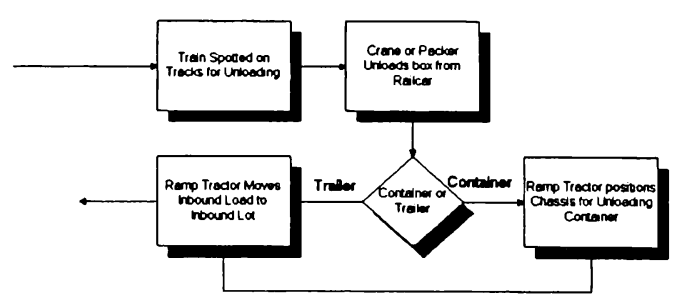

Figure 2: Unloading Process

The process arriving or departing trailers and containers under go are relatively simple. The complexity in the system occurs from the variation in traffic volumes handle and the customer demand for reliable timely transportation service.

\section{THE MODEL}

The Intermodal Capacity Planning model is written 
in SIMAN with user code written in C. Animation of the terminal is provided using CINEMA. The original model was written using SIMAN version IV, the current model has been converted to SIMAN and CINEMA V. The process description portion of the model uses SIMAN with $\mathrm{C}$ code performing the majority of the statistical, initialization and logic routines.

The Intermodal Capacity Planning Model provides a discrete event simulation of intermodal terminal operations. The model provides information on capacity related issues: equipment utilization; parking requirements; and train schedule performance.

The model simulates multiple one week scenarios with each daily occurrence of train schedules, gate arrivals repeated for each week during the run. Multiple weekly runs are necessary to account for the cyclic nature of workloads experienced at the terminal.

Changing the model for a new terminal requires the definition of the physical layout of the facility and the operational parameters: equipment quantities, schedules ,processing times and local operating practices.

The model is comprehensive and treats the overall facility as a single system. Major processes within the terminal system are modeled to reflect the interaction of sub-systems on overall terminal capacity.

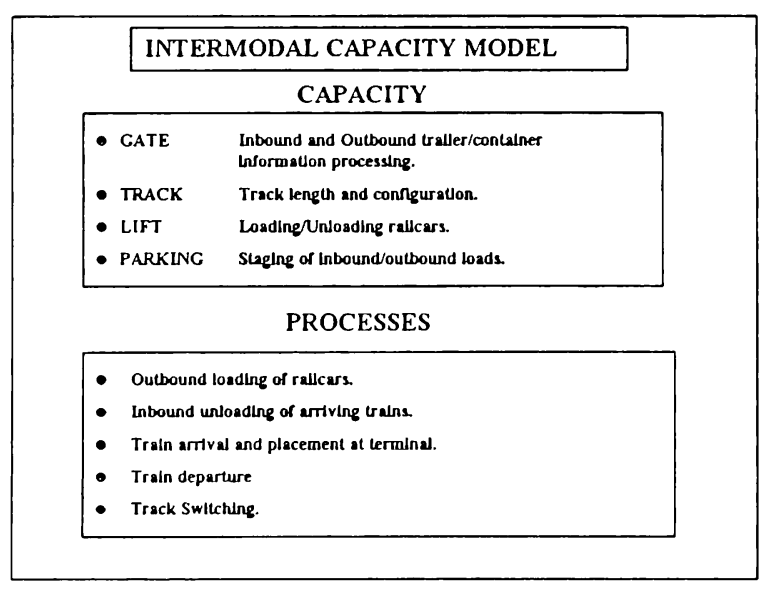

Figure 3: Terminal Capacity determined from interaction of all major processes

\subsection{Loading Railcars}

Loading of the railcar starts with the generation of the departing load entity. The data for generating the outbound load entity (departing on train) in the baseline model comes from historical information collected from on-line systems. The pertinent data includes the destination, type of box (container or trailer) size and the time it was processed at the gate. The gate arrival time is used to calculate the arrival rate by hour for each day of the week.

The outbound arrivals for the day are generated at the beginning of each day and stored in a link list. As entities enter the system the are removed from the list. The list is used to keep track of entities which have not yet entered the system, the load routine uses the list of remaining entities to perform a pseudo load which determines when a track is loaded to capacity. This method was used because of the large number of car loading configurations available and solved the problem of the model searching the track queues and finding when to switch the track queue.

Entities are used to represent both the inbound and outbound loads. An arriving entity delays at the gate for processing of waybill information and inspection of the equipment. The entity proceeds to a event routine to determine if it can be loaded on a railcar. The load routine checks an internal array showing which tracks have the load flag set, the track is then checked for a destination match with the departing entity. The next criteria for loading is the appropriate type of load configuration.

The load event searches the track queue looking for a railcar which is not fully loaded. The entity is then placed in an open well on the railcar, and the new configuration is checked against the load configuration array. If the entity cannot be loaded it is placed in a queue which is check at each change in track or load status, entities which can be loaded are allocated a slot on the railcar entity. The entity then requests a transporter to move it to the location on the facility where the railcar is located. The SIMAN transporter element is used to model the ramp tractor, a ramp tractor is responsible for moving loads between the parking areas and the loading tracks.

As mentioned earlier the load event routine for those entities which cannot be loaded are checked against a list of all the arrivals for the day. The load event performs a pseudo load to see if after each arrival a load configuration will exist allowing the entity to be loaded. When additional arrivals will not result in a successful load the track is pulled and spotted with empty railcars.

\subsection{Track Switching}

The track structure in terms of switches and track leads are not modeled. The model does approximate the track switching time and switch engine utilization 
by using a standard delay formula. The delay time calculation is a function of the number of cars being pulled on the track plus a standard movement delay for the switch engine. The track status is changed and the track status flag set to unavailable while the cars are pulled from the track. The track flag will keep the load routine from trying to allocate a slot on a railcar entity which is on the track being switched.

\subsection{Train Arrivals and Departures}

The train schedules are read into the model at the beginning of each run. This file controls the time of arrival, the size of the trains and the type of cars making up the train. Train arrival times are determined for each train based on a uniform distribution. The following list contains the information include in the train input file.

- Train Symbol.

- Train Identification Number.

- Minimum number of cars.

- Maximum number of cars.

- Arrival/Departure Day.

- Minimum Arrival/Departure time.

- Maximum Arrival/Departure time

- Train Cutoff (Departing Trains Only).

- Block (Departing Trains Only).

- Probability of Car types.

The train entity controls the number of cars and the car types which make up the train. The train entity is split into individual car entities. The size of the train is calculated as the individual cars are created. Articulated cars are treated as one car for the purpose of track allocation. The entities representing the articulated cars are split into individual car entities at the time the car enters the track queue. Track availability is evaluated to determine whether or not the train can enter the facility. Track assignment for a inbound train is based on a user define preferred order selection rule. The tracks are modeled as queues with a resource controlling the length of track available.

Car loading configurations are determined at the time a individual railcar is assigned to a track in the model. The model generates a type of load $(1=$ trailer, $2=$ container $)$ and the quantity on the railcar. The inbound entity is placed in a queue to request the crane transporter for unloading. The entity will also request a ramp tractor for moving the load to the parking area.

Train Departure processing is much simpler, the train entity acts only to remove the appropriate cars from the track queues. Outbound loaded cars are assigned a destination block at the time the load is placed on the car, this destination attribute is used to determine which cars to remove from the track queue. When a train is ready for departing, the model first checks to make sure that none of the cars have a allocated slot which has not been filled. Once all allocated slots have been filled the railcars are removed from the track queues.

\subsection{Unloading Inbound Trains}

The unloading of the inbound railcar is initiated when the railcar is placed in the track queue. The entity representing the inbound load is placed in a queue requesting the lift equipment. The load entity controls the movement of the crane transporter and the ramp tractor transporter.

SIMAN free path transporter constructs are used to model the lift equipment and the ramp tractors. The model uses free path transporters to avoid directional conflicts. Due to the number of stations defined for the facility layout the guided transporter system map is used to determine the next intersection/station routing for the free path transporter. This method allowed the model to generate the distance matrix.

Inbound trailers and containers remain on the facility until they are pickup by the customer. After placement of the load entity in a parking queue an additional entity is created and scheduled to return to the gate module representing a customer pickup .The customer arrival entity removes the entity from the parking queue and exits the facility. Historical information is used to determine the appropriate distribution of dwell times.

\subsection{Terminal Manger}

The Terminal Manager is a term given to the logic coded in the model to control the operations of the terminal. Modeling the activities of processing inbound and outbound trailers and containers, loading and unloading of trains is relatively straight forward. The difficulty occurs when trying to incorporate the logic a terminal manager would use in switching and assigning trains to yard tracks. Initially these rules were hard coded into the model, this approach worked but lacked the flexibility needed to make the model adaptable to other terminals and required coding changes during model verification. This limitation was also evident during the validation stage of model development. The Terminal Manager was designed to 
allow the rules to be easily changed during model validation and experimentation.

\begin{tabular}{|c|c|c|c|c|c|c|c|c|c|}
\hline Seq Evat & Track DS & Nent & & 100 & KI & EST & VAT & ION & COMMENTS \\
\hline $1 \mathrm{TU}$ & $3 T$ & $\mathrm{OB}$ & 1 & 0 & 0 & 0 & 0 & 0 & 0 IRaute to Realcar Slarage \\
\hline $2 \pi$ & $3 \mathrm{RY}$ & sw & 1 & 0 & 0 & 0 & 0 & 0 & 0 ILoodOBunits \\
\hline $3 \mathrm{SW}$ & $3-$ & DP & 0 & 0 & 0 & 0 & 0 & 0 & 0 ISwitch Tradk-Thainar \\
\hline $4 D P$ & $3-$ & - & 0 & 0 & 0 & 0 & 0 & 0 & 0 I Yard Nex bbard train \\
\hline $5 \mathrm{TU}$ & $2 \pi \mathrm{L}$ & $\mathrm{OB}$ & 1 & 0 & 0 & 0 & 0 & 0 & 0 lBlack Axigmus rdeas \\
\hline $6 \pi$ & $2 \mathrm{RY}$ & SW & 1 & 0 & 0 & 0 & 0 & 0 & 0 Thenistrack fill \%? \\
\hline $7 \mathrm{SW}$ & $2-$ & DP & 1 & 0 & 0 & 0 & 0 & 0 & 0 ISwitch at tradk 2 \\
\hline $8 \mathrm{DP}$ & $2-$ & - & 0 & 0 & 0 & 0 & 0 & 0 & 0 ! Yard Nex tband train \\
\hline $9 \mathrm{TU}$ & $1 \pi$ & $\mathrm{OB}$ & 1 & 0 & 0 & 0 & 0 & 0 & 0 llondatthands on track \\
\hline $10 \mathrm{TL}$ & $1 \mathrm{RY}$ & SW & 1 & 0 & 0 & 0 & 0 & 0 & 0 I Whenis tredk fill \%? \\
\hline II SW & $1-$ & DP & 0 & 0 & 0 & 0 & 0 & 0 & 0 ISwitch Tradk-TrainaI \\
\hline $12 \mathrm{DP}$ & $1-$ & - & 0 & 0 & 0 & 0 & 0 & 0 & 0 IYard Nex trbandtrain \\
\hline $13 \pi$ & $1 \mathrm{TL}$ & $O B$ & 2 & 0 & 0 & 0 & 0 & 0 & 0 | I look Assignerts reas \\
\hline
\end{tabular}

Figure 3. Terminal Manager input file

Table 1. Terminal Manager Codes and Event types

Event - Event completed in the model.

Track - Track event occurred on.

DS - Destination of cars after event completion

Next - Next event for track.

Block - Block destination loaded on track

Event types

TU - Railcars on track unloaded.

TL - Load cars with destination block.

SW - Switch cars on track

DP - Remove cars from track queue.

RY - Switch track to car storage.

\section{MODEL OUTPUTS}

The model uses the standard SIMAN output reports and custom report files to output simulation results. The SIMAN summary report displays the track utilization's, parking space requirements and queuing information. Lift equipment and ramper utilization reports are generated from event routines which tally hourly and daily statistics.

The critical part of Intermodal service is the ability to provide quality service. This service quality is based on train schedule performance. The model provides information on key areas of train schedule performance, the performance measures are based on the facilities ability to meet established schedules. They include:

- Train arrival time vs. train placement.
- Actual load grounding vs. planned grounding.

- Outbound cut-off time to train departure.

Failure to meet these performance measures indicates a potential capacity problem.

\section{AUTHOR BIOGRAPHY}

MICHAEL L. WEIGEL is a Senior Analyst in the Operations Analysis Group at Union Pacific Railroad. He has developed simulation models in the areas of rail transportation, aerospace logistics and manufacturing. $\mathrm{He}$ received a B.S. degree in Industrial Engineering from the University of Nebraska. He is a member of IIE. 